\title{
Biofilm Formation of Acinetobacter baumannii Under in vitro and in vivo Colistin Exposure
}

\author{
Berna Özer ${ }^{\mathbf{1}}$, Cansel Vatansever ${ }^{\mathbf{1}}$, Özlem Doğan ${ }^{\mathbf{1}}$, Şiran Keske ${ }^{\mathbf{2}}$, Önder Ergönü|${ }^{\mathbf{1}}$, Füsun Can ${ }^{\mathbf{1}}$ \\ 1 Department of Infectious Diseases and Clinical Microbiology, Koç University School of Medicine, İstanbul, Turkey \\ 2 Department of Infectious Diseases and Clinical Microbiology, VKV Amerikan Hospital, İstanbul, Turkey
}

\begin{abstract}
Objective: We aimed to find the alterations in biofilm formation of Acinetobacter baumannii (A. baumannii) during adaptation to colistin resistance under colistin stress.

Materials and Methods: Eighteen patients with an isolation of A. baumannii (nine colistin resistant and nine colistin susceptible) and additionally two patients that develop colistin resistant A. baumannii infection during hospital stay were included the study. For in vitro adaptation study, four colistin susceptible strains of one of the patients were sub-cultured onto Mueller Hinton agar containing sub-MIC concentrations of colistin for 40 serial passages. The colistin resistance was determined by broth dilution. Biofilm production was measured by crystal violet assay and images were taken with confocal microscopy. Genotyping of selected isolates was done by MLST. The thirty-three per cent of ColR A. baumannii was isolated from respiratory tract.

Results: The biofilm formation in ColR A. baumannii isolates was $78 \%$, and it was $54 \%$ in ColS A. baumannii. In the adaptation study, we did not find a difference in biofilm levels of laboratory-induced colistin resistant generations. On the other hand, clinical ColR isolate was found to be 2.6-3.4 times more biofilm producer than laboratory induced generations. Conclusion: We suggest that A. baumannii may develop adaptation mechanisms to constitute colistin-resistance in the presence of host-dependent factors and environmental stress conditions in order to gain stronger biofilm production to enhance virulence.
\end{abstract}

Keywords: Acinetobacter baumannii, colistin resistance, biofilm, adaptation, virulence

\section{INTRODUCTION}

A cinetobacter baumannii is one of the most pathogenic nosocomial infection agents because of its extreme resistance to almost all known antibiotics and host immune responses (1). The emergence of colistin-resistance in A. baumannii has been reported throughout the world $(2,3)$.

Biofilm formation ability enhances virulence of A. baumannii by increasing survival of cells in unfavourable environmental conditions, such as underexposure of disinfectants, antibiotics or attack of immune cells $(4,5)$. Antibiotic-resistant phenotypes

\section{Corresponding Author: Füsun Can \\ E-mail: \\ fucan@ku.edu.tr}

Received: March 4, 2019 Accepted: April 30, 2019 Published: May 30, 2019

Suggested citation: Özer B, Vatansever C, Doğan Ö, Keske Ş, Ergönül Ö, Can F. Biofilm Formation of Acinetobacter baumannii Under in vitro and in vivo Colistin Exposure. Infect Dis Clin Microbiol 2019; 1: 26-33.

DOI: 10.5152/idcm.2019.19004 
strongly influence biofilm forming capacity of A. baumannii (6). The expression of biofilm-associated virulence genes and biofilm thickness in MDR (multidrug-resistant) strain was found to be higher than drug-sensitive strain (7).

A. baumannii acquires colistin resistance by rapid induction of resistance mechanisms in the presence of colistin (8). Simultaneously, bacteria display an adaptation to colistin exposure with significant changes in transcriptome profile and cell membrane structure (9). Biofilm-related outer membrane lipoprotein (pgaB) was found to be upregulated in colistin resistant strains (10). However, another study showed that mutations involved in colistin resistance phenotype downregulate the expression of biofilm-associated genes of colistin-resistant isolates (11).

The understanding of bacterial pathogenesis has lead to the development of many potential strategies and novel drugs to treat MDR bacteria. Anti-virulence treatment is one of the promising therapy approaches (12). The aim of this study is to explore the alterations in biofilm formation capacity of $\mathrm{A}$. baumannii during adaptation to colistin resistance under colistin stress.

\section{MATERIALS AND METHODS}

\section{Study population and bacterial strains}

We included 18 patients with isolation of A. baumannii between October 2014 and September 2018 from different centres in Turkey. The gender, age, source, carbapenem resistance, carbapenemase type, colistin exposure, and colistin resistance data were recorded. For all patients one representative A. baumannii (nine colistin resistant and

\section{HIGHLIGHTS}

- Colistin resistant Acinetobacter baumannii has increased biofilm production capacity.

- The biofilm production might be a part of adaptation response of bacteria to colistin resistance. nine colistin susceptible) isolate was included. Additionally, we selected two patients that developed colistin resistant A. baumannii infection during their hospital stay. The colistin susceptible and resistant pairs of A. baumannii isolated during the patients' hospital course were studied.

\section{Antibiotic susceptibility testing}

Colistin minimum inhibitory concentrations (MICs) were determined by broth microdilution method. The isolates were grown on Tryptic Soy Agar (TSA) (Becton, Dickinson, U.S.) overnight. Then, the turbidity of each isolate was adjusted to 0.5 MacFarland by cation-adjusted Mueller Hinton broth (MH) using nephelometer (Becton, Dickinson, U.S.). Serial dilution was performed with final concentrations between $256 \mu \mathrm{g} / \mathrm{mL}$ and $0.25 \mathrm{mg} / \mathrm{L}$. The samples were incubated at $37^{\circ} \mathrm{C}$ overnight and MICs were determined by measuring absorbance values at $540 \mathrm{~nm}$ in addition to reading by the naked eye. Escherichia coli ATCC 25922 standard strain was used as a reference, and resistance breakpoint was set as $>2 \mu \mathrm{g} / \mathrm{mL}$ based on Clinical and Laboratory Standards Institute (CLSI) guideline (13).

\section{Assessment of biofilm formation}

Biofilm production was measured by Crystal Violet Assay. Thus, all isolates were grown on TSA overnight. Then, a single colony was inoculated into $5 \mathrm{~mL}$ of Tryptic Soy Broth (TSB) and incubated at $37^{\circ} \mathrm{C}$ on a shaker $(125 \mathrm{rpm})$ until turbidity reached 10-13 MacFarland. The cultures were diluted to 1:50 using TSB containing $0.1 \%$ glucose. $100 \mu \mathrm{L}$ of diluted cultures in 96 -well plate was incubated at $37^{\circ} \mathrm{C}$ for $24 \mathrm{~h}$. After removal of non-adherent bacteria, adherent bacteria were fixed by incubation at $60^{\circ} \mathrm{C}$ for $40 \mathrm{~min}$ and stained with $125 \mu \mathrm{L}$ of crystal violet. Bound crystal violet was dissolved by $95 \%$ ethanol. Optic Density (OD) values of wells were measured at $540 \mathrm{~nm}$. The isolates with ODs between 0.12> and $£ 0.5$ were considered weak, $>0.5$ were strong biofilm producers (14).

For imaging of biofilms, bacterial cells were fixed with 3.5\% formaldehyde solution overnight. The fixed cells were stained using the Live/Dead Backlight viability kit with following the manufacturer's protocol. Samples were examined with Leica DMI8 laser scanning confocal microscope $(15,16)$. 
Table 1. The demographic and laboratory data of study population.

\begin{tabular}{|c|c|c|c|c|}
\hline & & $\begin{array}{c}\text { Total } \\
\mathrm{n}=18(\%)\end{array}$ & $\begin{array}{l}\text { Colistin resistant } \\
\qquad(n=9)(\%)\end{array}$ & $\begin{array}{l}\text { Colistin susceptible } \\
\qquad(n=9)(\%)\end{array}$ \\
\hline \multicolumn{2}{|c|}{ Median age (min-max) } & $71(51-84)$ & $74(51-84)$ & $77(57-82)$ \\
\hline \multicolumn{2}{|c|}{ Female gender } & $11(61.1)$ & $8(88.9)$ & $3(33.3)$ \\
\hline \multirow{4}{*}{ Source } & Blood & $12(67)$ & $3(33)$ & $9(100)$ \\
\hline & Respiratory tract & $3(17)$ & $3(33)$ & - \\
\hline & Wound & $1(6)$ & $1(11)$ & - \\
\hline & Rectal swap & $2(11)$ & $2(22)$ & - \\
\hline \multicolumn{2}{|c|}{ Carbapenamase resistance } & $18(100)$ & $9(100)$ & $9(100)$ \\
\hline \multicolumn{2}{|c|}{ OXA-23 Carbapenamase type } & $18(100)$ & $9(100)$ & $9(100)$ \\
\hline \multicolumn{2}{|c|}{ Colistin exposure } & $15(83)$ & $9(100)$ & $6(67)$ \\
\hline
\end{tabular}

Table 2. The overview of ColR-ColS A. baumannii pairs isolated from two patients.

\begin{tabular}{|c|c|c|c|c|c|c|}
\hline Patient No & Isolate & Colistin Resistance & $\begin{array}{c}\text { Colistin MIC } \\
\text { (mg/L) }\end{array}$ & $\begin{array}{c}\text { Duration of colistin } \\
\text { therapy at the day } \\
\text { of isolation }\end{array}$ & ST Type & Source \\
\hline \multirow{5}{*}{1} & K411 & $\mathrm{S}$ & 1 & 0 & ST2 & Sputum \\
\hline & K412 & $\mathrm{S}$ & 1 & 0 & ST2 & $\mathrm{IAF}^{*}$ \\
\hline & K408 & $\mathrm{S}$ & 0.5 & 9 & ST2 & $\mathrm{IAF}$ \\
\hline & K399 & $\mathrm{S}$ & 1 & 15 & ST2 & Sputum \\
\hline & K409 & $\mathrm{R}$ & 16 & 25 & ST2 & $\mathrm{IAF}$ \\
\hline \multirow{2}{*}{2} & K1007 & $\mathrm{S}$ & 2 & 0 & ST2 & Respiratory tract \\
\hline & K1006 & $\mathrm{R}$ & 16 & 4 & $\mathrm{ST} 2$ & Respiratory tract \\
\hline
\end{tabular}

IAF*: Intra abdominal fluid; S: susceptible; R: resistant

\section{In vitro adaptation study}

For in vitro adaptation, four colistin susceptible strains (K399, K408, K411 and K412) of one of the patients with both colistin resistant and susceptible A. baumannii isolation were selected. The selected susceptible isolates were sub-cultured onto Mueller Hinton agar (MHA) containing $1 \mu \mathrm{g} / \mathrm{mL}$ colistin (Sig- ma) by 40 serial passages. Biofilm production and colistin MICs of each generation were determined.

\section{Genotyping of isolates}

MLST was performed by amplifying seven housekeeping genes, namely, cpn60, fusA, gltA, pyrG, recA, rplB, and $r p o B$ according to the protocol developed 
by University of Oxford on A. baumannii MLST website (https://pubmlst.org/abaumannii/). Allelic profiles and sequence types (STs) were determined using Applied Math Bionumerics V7.6 software.

\section{RESULTS}

Among 18 patients, seven of them were male. All of the patients with colistin resistant A. baumannii isolation received colistin therapy. Twelve of the isolates $(67 \%)$ were obtained from blood cultures. All isolates were carbapenem resistant and OXA-23 producers (Table 1).

Data of the two patients that develop colistin resistant $A$. baumannii infection during hospital stay was presented in Table 2. Both patients received colistin therapy, and the patient
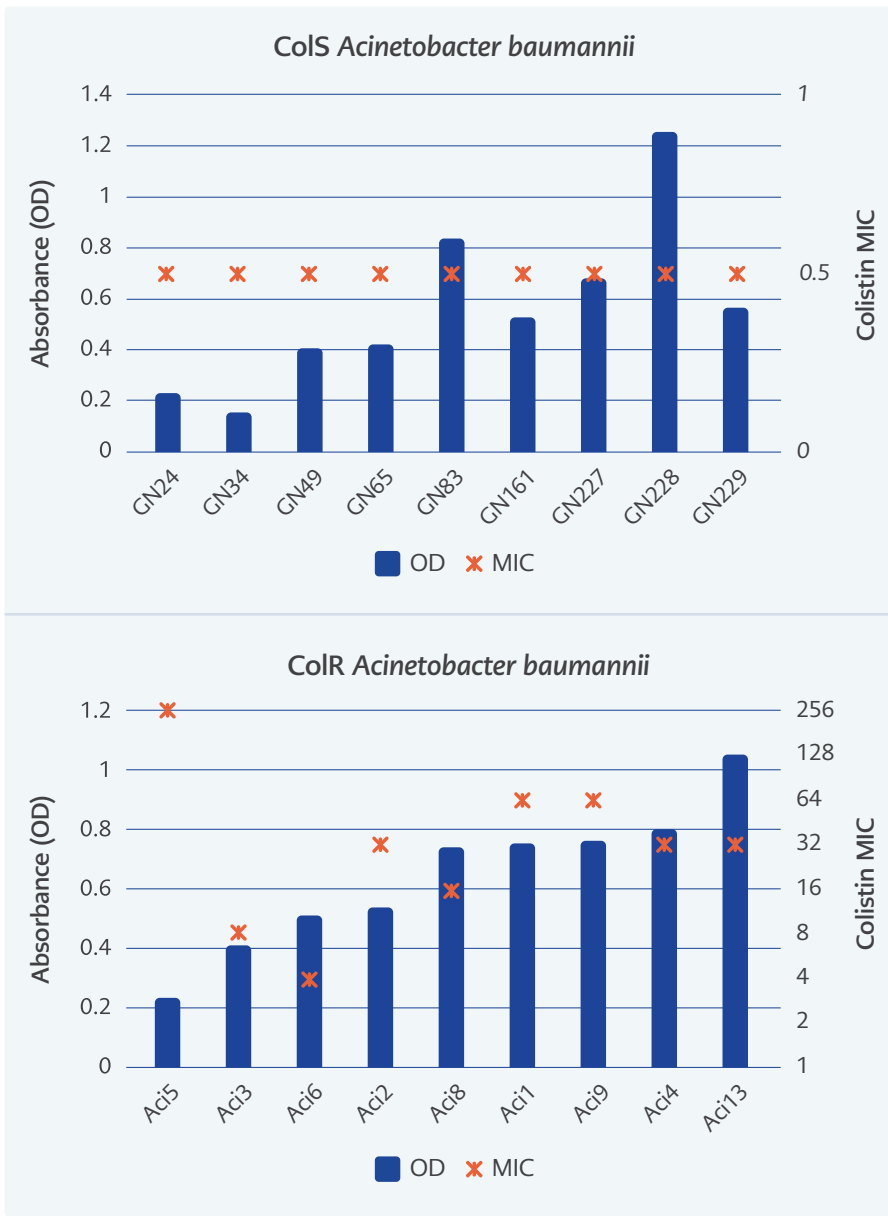

Figure 1. The biofilm and MIC levels of ColS and ColR A. baumannii isolates. OD: Optic Density; MIC: Minimum inhibitory concentration.
2 died after four days of A. baumannii isolation. All isolates belonged to global ST2 clone.

\section{Biofilm formation of A. baumannii isolates}

The biofilm production of ColR and Cols isolates were shown in Figure 1. Among all A. baumannii, $54 \%$ (5/9) of ColS isolates and 78\% of ColR (7/9) were found to be strong biofilm producers. The median of OD values for Cols isolates was 0.53 (0.23-1.24), median for ColR isolates was 0.73 (0.12-1.05). In seven of the nine ColR isolates, the elevated colistin MICs were found to be accompanied to high levels of biofilms.

The biofilms and MICVs of A. baumannii from two selected patients were shown in Figure 2. In patient 1, biofilm production of ColR isolate was higher than ColS original strain; however, a slight decrease was observed in biofilm production of ColR strain than Cols counterpart in patient 2 (Figure 2A). Also, microscope images showed that biofilm productivity of ColR strain was stronger than Cols in patient 1 , but there was a decrease in biofilm formation of ColR isolate compared to Cols strain in patient 2. These results were consistent with the crystal violet results.

Biofilm production of $A$. baumannii generations during in vitro adaptation to colistin exposure The biofilm production and colistin MICs of selected generations of four colistin susceptible isolates (K399, K408, K411, and K412) and ColR isolate (K409) from patient 1 were shown in Figure 3. The Colistin MICs were increased to resistance breakpoint $(2 \mathrm{mg} / \mathrm{mL}$ ) at the first generation.

The results showed no considerable difference in biofilm formation between the generations. The K409 ColR clinical isolate had 2.6-3.4 fold stronger biofilm production capacity than laboratory induced colistin resistant generations.

\section{DISCUSSION}

The emergence of colistin resistance in A. baumannii has increased the need for the development of new therapeutic approaches. Inhibition of virulence factors is becoming one of the most popular strategies for the treatment of infections $(12,17)$. Biofilm for- 
mation of A. baumannii is a significant virulence determinant that because of the strong interaction of bacteria with host cells (7).

Here we presented 18 cases with an isolation of A. baumannii from different specimens. $17 \%$ of total isolates and $33 \%$ of ColR isolates were from respiratory specimens. The biofilm production rate of all isolates was $67 \%$. In 2008, a multicentered cohort study showed $63 \%$ biofilm formation among $\mathrm{A}$. baumannii isolated from various sources. They also reported that respiratory isolation was associated with non-biofilm production (18). Controversially, in another study, high-level biofilm formation was observed in respiratory specimens of 61 patients in 2013 (19).

In this study, we detected strong biofilm production (OD>0.5) in $78 \%$ of ColR, and in $54 \%$ of Cols A. baumannii isolates. Recent studies reported a significant association between high biofilm formation capacity and multidrug-resistant profile of A. baumannii (20). A multi-centric hospital based study showed that more than $90 \%$ of the biofilm producer A. baumannii isolates were multidrug-resistant (6). Additionally, MDR and biofilm producer A. baumannii strains were reported from outbreaks in hospitals especially in intensive care units $(21,22)$. However, there are controversial reports about the colistin resistance with biofilm production. Farshadzadeh et al. (11) found that the biofilm-forming ability of ColR A. baumannii was not significantly different from their Cols counterparts. They claimed that biofilm formation capacity could be related to the change in growth rate. In two different studies, Pournaras and Dafopoulou revealed that the acquisition of colistin resistance via a single pmrB mutation was associated with an impaired biofilm formation capacity because of the decrease in growth rate $(23,24)$. Another important finding of our study was the association of high colistin MICs with high biofilm values among ColR A. baumannii. A very re-

Figure 2. The biofilm and MIC levels of ColS-ColR A. baumannii pairs from two selected patients. Crystal violet assay and MICS (A); confocal images of biofilms (B). In confocal images, red color indicates dead, green color indicates live cells. OD: Optic Density; MIC: Minimum inhibitory concentration.

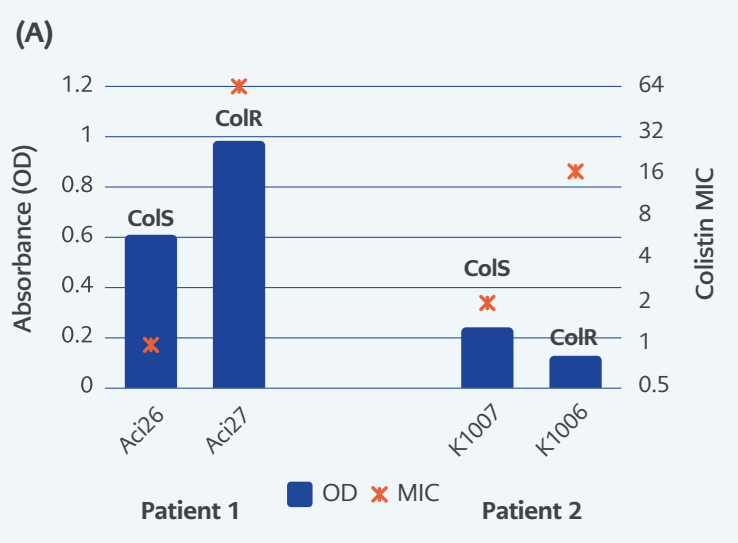

(B)
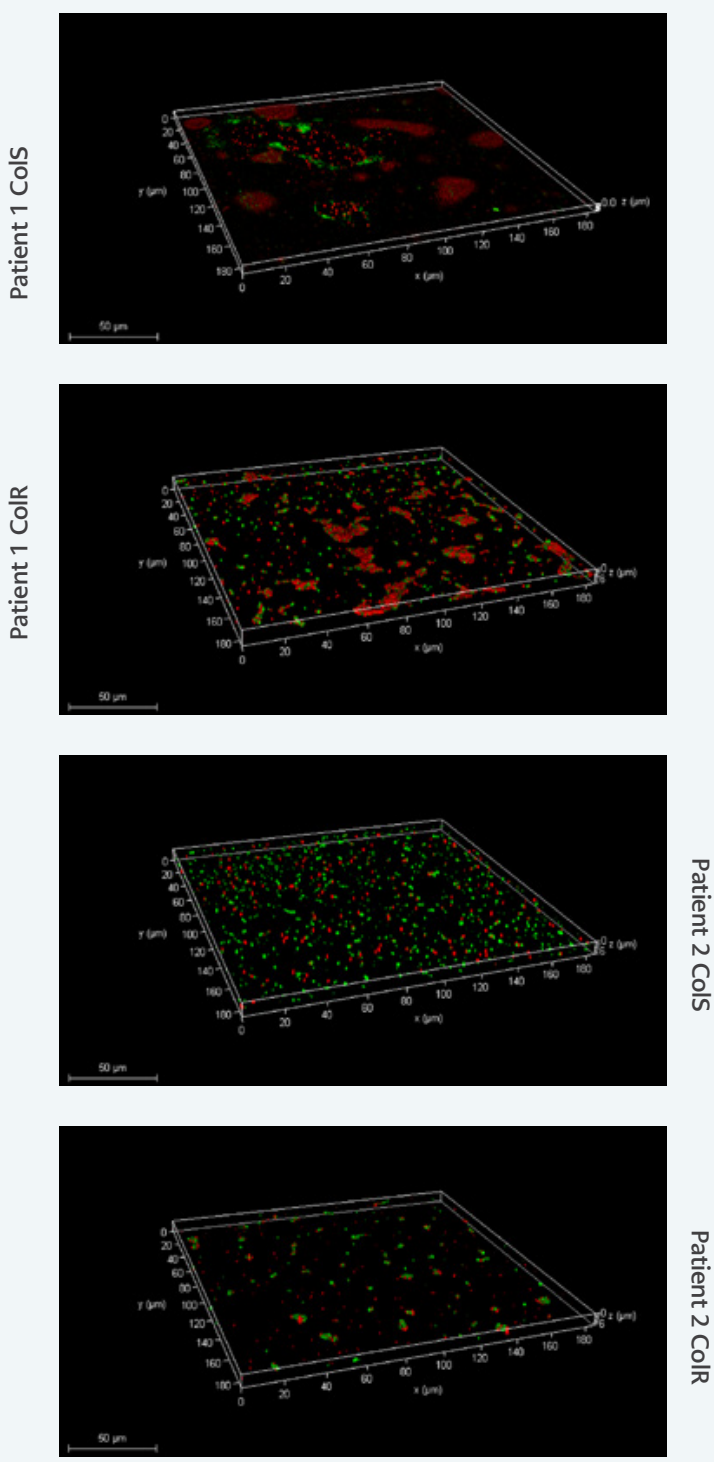

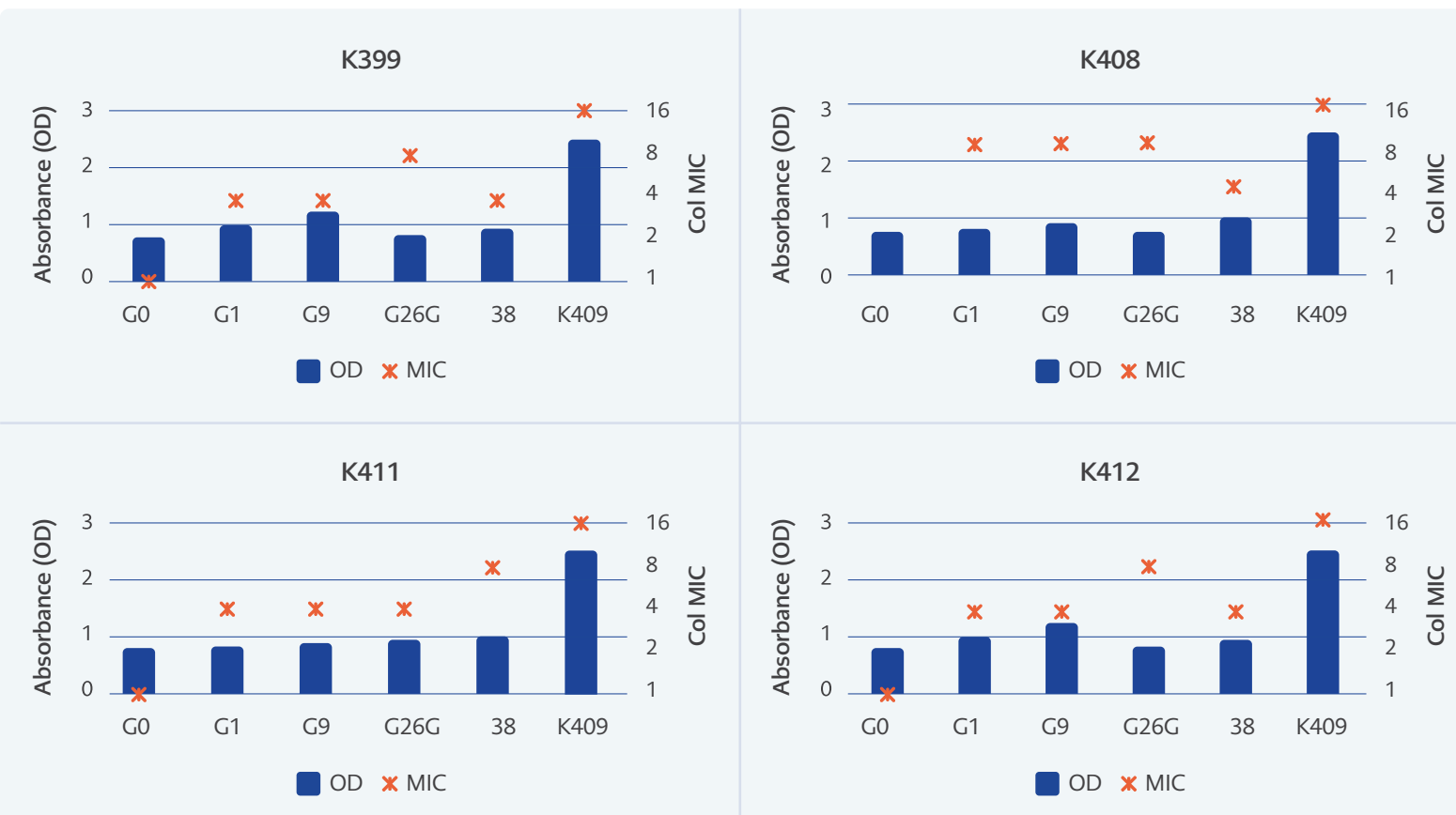

Figure 3. Biofilm production and colistin MICs of selected generations during in vitro adaptation to colistin exposure. OD: Optic Density; MIC: Minimum inhibitory concentration. K399, K408, K411 and K412 are the numbers of each isolates.

cent study reported overexpression of biofilm-associated genes underexposure of colistin (25). In our study, all patients received colistin therapy before isolation of ColR strains.

In this study, we also examined the alterations in biofilm ability of A. baumannii during the in vitro adaptation period to colistin resistance and compared the in vivo and in vitro results. The ColR (K409) isolate was detected on the $25^{\text {th }}$ day of the colistin therapy. This isolate was found to be a strong biofilm producer revealed 2.6-3.4 times more biofilm levels than Cols first pair. When we mimicked in vivo colistin use in the laboratory, the exposure of subMIC concentration of colistin $(1 \mathrm{mg} / \mathrm{L})$ did not alter biofilm production of the generations. Even the 25th generation that corresponds to the duration of colistin therapy at the isolation day of clinical ColR A. baumannii did not show a difference than Cols first pair. These results suggested that colistin exposure is not the only factor that affects the in vivo biofilm formation ability of A. baumannii under colistin stress. Other contributors might have a role in biofilm production of bacteria during the development of colistin resistance. Similarly, a study published in 2018 declared that colistin exposure did not have an effect on the biofilm-forming capacity of laboratory-induced A. baumannii (26). Besides, recent in vitro studies reported the biological cost of adaptation to colistin resistance in A. baumannii. The loss of LPS leads fitness cost and low biofilm formation capacity so consequently reduces virulence $(11,27,28)$.

In conclusion, the biofilm forming capacity of ColR A. baumannii isolates is higher than Cols A. baumannii. On the other hand, we did not find a difference in biofilm levels of laboratory-induced colistin resistant generations. We suggest that A. baumannii may develop adaptation mechanisms to constitute colistin-resistance in the presence of host-dependent factors and environmental stress conditions in order to gain stronger biofilm production capacity to enhance virulence. 
Ethics Committee Approval: The regulation on clinical research entered into force on 19 August 2011 (Official Gazette number 28030) in Turkey and ethics committees within the scope of this regulation began to be formed thereafter. Present study was conducted before related regulation and therefore ethics committee approval was not provided.

Informed Consent: Written informed consent was obtained after explaining to them the details of our study from the patients who participated in this study.

Peer-review: Externally peer-reviewed
Author Contributions: Concept - B.Ö., F.C., Ö.D.; Supervision - F.C., Ö.E.; Resources - Ş.K., Ö.E., C.V.; Materials - B.Ö., C.V.; Data Collection and/or Processing - B.Ö., C.V.; Analysis and/or Interpretation - B.Ö., Ö.D., C.V.; Literature Search - B.Ö., Ö.D.; Writing Manuscript - B.Ö., Ö.D.; Critical Reviews - F.C., Ö.E.

Conflict of Interest: The authors have no conflict of interest to declare.

Financial Disclosure: The authors declared that this study has received no financial support.

\section{REFERENCES}

1 Aydin M, Ergonul O, Azap A, Bilgin H, Aydin G, Cavus SA, et al. Rapid emergence of colistin resistance and its impact on fatality among healthcare-associated infections. J Hosp Infect 2018; 98: 260-3.

2 Gales AC, Jones RN, Sader HS. Contemporary activity of colistin and polymyxin B against a worldwide collection of Gram-negative pathogens: results from the SENTRY Antimicrobial Surveillance Program (2006-09). J Antimicrob Chemother 2011; 66: 2070-4.

3 Ergonul O, Aydin M, Azap A, Basaran S, Tekin S, Kaya S, et al. Healthcare-associated Gram-negative bloodstream infections: antibiotic resistance and predictors of mortality. J Hosp Infect 2016; 94: 381-5.

4 Perez F, Hujer AM, Hujer KM, Decker BK, Rather PN, Bonomo RA. Global challenge of multidrug-resistant Acinetobacter baumannii. Antimicrob Agents Chemother 2007; 51: 3471-84.

5 Wong D, Nielsen TB, Bonomo RA, Pantapalangkoor P, Luna B, Spellberg B. Clinical and Pathophysiological Overview of Acinetobacter Infections: a Century of Challenges. Clin Microbiol Rev 2017; 30: 409-47.

6 Ebrahim Babapour AH, Reza Mirnejad, Seyed-Abdolhamid Angaji, Nour, Amirmozafari. Biofilm formation in clinical isolates of nosocomial Acinetobacter baumannii and its relationship with multidrug resistance. Asian Pac J Trop Biomed 2016.

7 Eze EC, Chenia HY, El Zowalaty ME. Acinetobacter baumannii biofilms: effects of physicochemical factors, virulence, antibiotic resistance determinants, gene regulation, and future antimicrobial treatments. Infect Drug Resist 2018; 11: 2277-99.

8 Olaitan AO, Morand S, Rolain JM. Mechanisms of polymyxin resistance: acquired and intrinsic resistance in bacteria. Front Microbiol 2014; 5: 643.

9 Chung ES, Ko KS. Eradication of persister cells of Acinetobacter baumannii through combination of colistin and amikacin antibiotics. J Antimicrob Chemother 2019.

10 Cafiso V, Stracquadanio S, Lo Verde F, Gabriele G, Mezzatesta ML, Caio C, et al. Colistin Resistant A. baumannii: Genomic and Transcriptomic Traits Acquired Under Colistin Therapy. Front Microbiol 2018; 9: 3195.
11 Farshadzadeh Z, Taheri B, Rahimi S, Shoja S, Pourhajibagher M, Haghighi MA, et al. Growth Rate and Biofilm Formation Ability of Clinical and Laboratory-Evolved Colistin-Resistant Strains of Acinetobacter baumannii. Front Microbiol 2018; 9: 153.

12 Muhlen S, Dersch P. Anti-virulence Strategies to Target Bacterial Infections. Curr Top Microbiol Immunol 2016; 398: 147-83.

13 Clinical and Laboratory Standards Institute (2010) Performance standards for antimicrobial susceptibility testing: 20th informational supplement. CLSI, Wayne.

14 Tsikrikonis G, Maniatis AN, Labrou M, Ntokou E, Michail G, Daponte A, et al. Differences in biofilm formation and virulence factors between clinical and fecal enterococcal isolates of human and animal origin. Microb Pathog 2012; 52: 336-43.

15 Schlafer S, Meyer RL. Confocal microscopy imaging of the biofilm matrix. J Microbiol Methods 2017; 138: 50-9.

16 Ramstedt M, Ribeiro IAC, Bujdakova H, Mergulhao FJM, Jordao L, Thomsen P, et al. Evaluating Efficacy of Antimicrobial and Antifouling Materials for Urinary Tract Medical Devices: Challenges and Recommendations. Macromol Biosci 2019: e1800384.

17 Rasko DA, Sperandio V. Anti-virulence strategies to combat bacteria-mediated disease. Nat Rev Drug Discov 2010; 9: 11728.

18 Rodriguez-Bano J, Marti S, Soto S, Fernandez-Cuenca F, Cisneros JM, Pachon J, et al. Biofilm formation in Acinetobacter baumannii: associated features and clinical implications. Clin Microbiol Infect 2008; 14: 276-8.

19 Kaliterna V, Goic-Barisic I. The ability of biofilm formation in clinical isolates of Acinetobacter baumannii belonging to two different European clones causing outbreaks in the Split University Hospital, Croatia. J Chemother 2013; 25: 60-2.

20 Bardbari AM, Arabestani MR, Karami M, Keramat F, Alikhani MY, Bagheri KP. Correlation between ability of biofilm formation with their responsible genes and MDR patterns in clinical and environmental Acinetobacter baumannii isolates. Microb Pathog 2017; 108: 122-8.

21 Manchanda V, Sanchaita S, Singh N. Multidrug resistant acinetobacter. J Glob Infect Dis 2010; 2: 291-304. 
22 Eijkelkamp BA, Stroeher UH, Hassan KA, Paulsen IT, Brown MH Comparative analysis of surface-exposed virulence factors of Acinetobacter baumannii. BMC Genomics 2014; 15: 1020.

23 Pournaras S, Poulou A, Dafopoulou K, Chabane YN, Kristo I, Makris D, et al. Growth retardation, reduced invasiveness, and impaired colistin-mediated cell death associated with colistin resistance development in Acinetobacter baumannii. Antimicrob Agents Chemother 2014; 58: 828-32.

24 Dafopoulou K, Xavier BB, Hotterbeekx A, Janssens L, Lammens C, De E, et al. Colistin-Resistant Acinetobacter baumannii Clinical Strains with Deficient Biofilm Formation. Antimicrob Agents Chemother 2015; 60: 1892-5.

25 Sato Y, Unno Y, Ubagai T, Ono Y. Sub-minimum inhibitory concentrations of colistin and polymyxin B promote Acinetobacter baumannii biofilm formation. PLoS One 2018; 13: e0194556.
26 Bogdan M, Drenjancevic D, Harsanji Drenjancevic I, Bedenic B, Zujic Atalic V, Talapko J, et al. In vitro effect of subminimal inhibitory concentrations of antibiotics on the biofilm formation ability of Acinetobacter baumannii clinical isolates. J Chemother 2018; 30: 16-24.

27 Lopez-Rojas R, McConnell MJ, Jimenez-Mejias ME, Dominguez-Herrera J, Fernandez-Cuenca F, Pachon J. Colistin resistance in a clinical Acinetobacter baumannii strain appearing after colistin treatment: effect on virulence and bacterial fitness. Antimicrob Agents Chemother 2013; 57: 4587-9.

28 Beceiro A, Moreno A, Fernandez N, Vallejo JA, Aranda J, Adler B, et al. Biological cost of different mechanisms of colistin resistance and their impact on virulence in Acinetobacter baumannii. Antimicrob Agents Chemother 2014; 58: 518-26. 